\title{
Upper Bounds for the Critical Car Densities in Traffic Flow Problems
}

\author{
H. F. Chau \\ School of Natural Sciences, Institute for Advanced Study, \\ Olden Lane, Princeton, NJ 08540, USA \\ P. M. Hui and Y. F. Woo \\ Department of Physics, The Chinese University of Hong Kong, \\ Shatin, New Territories, Hong Kong
}

(June 30, 1995)

\begin{abstract}
In most models of traffic flow, the car density $p$ is the only free parameter in determining the average car velocity $\langle v\rangle$. The critical car density $p_{c}$, which is defined to be the car density separating the jamming phase (with $\langle v\rangle=0$ ) and the moving phase (with $\langle v\rangle>0$ ), is an important physical quantity to investigate. By means of simple statistical argument, we show that $p_{c}<1$ for the Biham-Middleton-Levine model of traffic flow in two or higher spatial dimensions. In particular, we show that $p_{c} \leq 11 / 12$ in 2 dimension and $p_{c} \leq 1-\left(\frac{D-1}{2 D}\right)^{D}$ in $D(D>2)$ dimensions.

Keywords: Biham-Middleton-Levine Model, Car Jamming Phase, Cellular Automata, Critical Car Density, Traffic Flow

PACS numbers: 89.40.+k, 05.60.+w, 64.60.Ak, 89.50.+r
\end{abstract}

Typeset using REVTEX 
Recently, there has been much interest in the study of traffic flow problems within the context of cellular automaton (CA) models. This approach has the advantages of being simple and computationally less intensive. Nagel and Schreckenberg [1] introduced a stochastic discrete automaton model in one dimension (1D) to study the transition from laminar traffic flow to start-to-stop-waves as the car density increases. Variations of the 1D model have been extensively studied [2 5]. Biham, Middleton, and Levine [6] (henceforth referred to as BML) introduced a simple two dimensional (2D) CA model with traffic lights and studied the average velocity of cars as a function of their concentration. In the BML model, each site on a 2D square lattice may take on an east-bound car (i.e., a car traveling in positive x-direction) and a north-bound car (i.e., a car traveling in positive $\mathrm{y}$-direction) both with probability $p / 2$. Otherwise, the site is empty with probability $1-p$. The probability $p$ thus corresponds to the car density. Each east-bound car moves at even time steps by one site provided that the neighboring site to the east is empty. Similarly, each northbound car moves at odd time steps by one site provided that the neighboring site to the north is empty. BML found that, using numerical simulations, the average car velocity in the long time limit vanishes when the car density is higher than a critical value $p_{c}$. The BML model has been generalized [0 10], for example, to include effects of two-level crossing (overpasses), faulty traffic lights, accidents, and anisotropy in car densities in east and north bound directions. Generalization of the model to other spatial dimensions is straight forward. "Traffic" models in higher dimensions, though may not be relevant to cars on the road, may be related to problems in information network and telecommunication.

Nagatani [7, 8] has proposed a mean field theory for the average car velocity. The theory has recently been criticized by Ishibashi and Fukui [11. They suggested that no complete traffic jam occurs unless $p=1$, in contrast to simulation results and other mean field approximations. They also speculated that the values of $p_{c}$ obtained in numerical experiments may be results of finite size effects and the use of periodic boundary conditions [11]. It is therefore desirable to derive bounds for $p_{c}$ in various spatial dimensions. In particular, if the upper bound of $p_{c}$ is less than unity, then there exists a range of car densities corresponding to a complete traffic jam. In what follows, we argue that there exists an upper bound for $p_{c}$ less than unity whenever the spatial dimension of the lattice is greater than one. In $2 \mathrm{D}$, we have $p_{c} \leq 11 / 12$ and we also give upper bounds for $p_{c}$ for spatial dimensions $D>2$.

Consider the BML traffic flow problem on a 2D square lattice. Instead of looking at how the traffic flows, we concentrate on how the traffic jams. We note that the $2 \mathrm{D}$ square lattice can be partitioned, for example, by a collection of inverted-L-shaped elements as shown in Fig. 1. Each element consists of 3 sites. For example, the element at the site $(i, j)$ contains a collection of three sites $L_{i, j} \equiv\{(i, j),(i-1, j),(i, j-1)\}$. Thus, we can partition the 2 D lattice by $\left\{L_{3 i+j, j}: i, j \in \mathbb{Z}\right\}$ (see Fig.1). Obviously, this is not the only way to partition the lattice. As we shall see, different partitions may lead to different upper bounds. It is, however, suffice for our purpose to consider this simple partition. The important point is that the elements can fill the underlying lattice with a density of non-zero measure.

Consider the case of a full lattice of cars, i.e., $p=1$, which trivially corresponds to a jamming phase. Consider, say, the element at $(i, j)$. The car at site $(i, j)$ can be removed without changing the system to a moving phase if the cars at sites $(i-1, j)$ and $(i, j-1)$ are north-bound and east-bound respectively. Assume the cars to have equal probability of being east or north bound, the probability of having the car at the middle of the inverted-L-shaped element removed without changing the system to a moving phase is $(1 / 2)^{2}$. Since each of the inverted-L-shaped element contains three 
sites, so in the limit of infinite system size, we can almost surely remove $(1 / 3) \times(1 / 2)^{2}=1 / 12$ of the cars in the system for any initial configuration of a full lattice of cars without changing the system from a jamming phase to a moving phase. This suggests an upper bound of $p_{c} \leq 11 / 12$ in 2D. Conversely, let us consider the case for an arbitrary initial configuration with car concentration $p=11 / 12$. A car moves into an empty site $(i, j)$ in the next time step or two whenever there is a north-bound car located at $(i, j-1)$ or an east-bound car located at $(i-1, j)$ at the present moment. The probability for this to occur is given by $q=\frac{11}{12} \frac{1}{2}+\frac{11}{12} \frac{1}{2}-\left(\frac{11}{12} \frac{1}{2}\right)^{2}=\frac{11}{12} \times \frac{37}{48}$. Therefore, in the mean field approximation, the expected number of sites at a given empty site $(i, j)$ can move is given by

$$
\begin{aligned}
\langle n\rangle & \approx(1-q) \times 0+q(1-q) \times 1+q^{2}(1-q) \times 2+\cdots+q^{k}(1-q) \times k+\cdots \\
& =\frac{q}{1-q} .
\end{aligned}
$$

So on the average, an empty site will be "blocked" after moving over $\langle n\rangle \approx 407 / 169 \approx 2.4$ sites. This is less than the typical separation between two empty sites (which equals $\sqrt{12} \approx 3.4$ ). Therefore, it is almost certain that a system with car concentration $p=11 / 12$ will evolve eventually to a jamming state. Our result should be contrasted with that of [11], in which it was found that complete jam occurs only when $p=1$. We believe that the discrepancy is due to the fact that Ishibashi and Fukui have incorrectly assumed that all car configurations are independent of each other. The upper bound on $p_{c}$ that we obtained here is consistent with all the numerical experiments (Biham et al. found that $p_{c} \approx 0.32$ for $512 \times 512$ lattice [6], while a finite size scaling analysis by Nagatani showed that $\left.p_{c}=0.42[7]\right)$. We also note that our argument above is true for any boundary conditions. And a more careful choice of partitions and elimination procedure may lead to a tighter bound [12].

Our argument can be readily generalized to anisotropic distribution of north and east bound cars as long as the traffic problem is not decoupled into independent $1 \mathrm{D}$ lanes. In $1 \mathrm{D}$, it is known that complete traffic jam only occurs when $p=1$ [6]. Let $q_{e}\left(q_{n}\right)$ be the probability of finding an east-bound (north-bound) car at a site given that there is a car at the site. We have $q_{e}+q_{n}=1$, and the ratio $q_{e} / q_{n}$ reflects the anisotropy. In this case, our argument leads to a bound in $2 \mathrm{D}$

$$
p_{c} \leq 1-\frac{q_{e} q_{n}}{3}
$$

Even in the 1D limit, either $q_{e}$ or $q_{n}$ vanishes and $p_{c} \leq 1$, which is consistent with the known results $p_{c}=1$.

Our argument can be carried over to any spatial dimension $D>2$. A naive extension of the above partition to 3D may be that of introducing elements with four sites. For example, the element $L_{i j k}$ at site $(i, j, k)$ contains the collection of sites $L_{i j k} \equiv\{(i, j, k),(i-1, j, k),(i, j-1, k),(i, j, k-1)\}$. However, one cannot partition a $3 \mathrm{D}$ cubic lattice with elements $L_{i j k}$. Since the choice of partition is quite arbitrary, we choose, for simplicity, to fill the underlying lattice with hypercubic elements in $D$ dimension, each of which contains $2^{D}$ sites. Consider a full lattice of cars. The probability of removing a car from a hypercube without changing the jamming character is $\left(1-\frac{1}{D}\right)^{D}$. Thus for $D>2$, we have an upper bound for $p_{c}$ given by

$$
p_{c} \leq 1-\frac{1}{2^{D}}\left(1-\frac{1}{D}\right)^{D}=1-\left(\frac{D-1}{2 D}\right)^{D} \text {. }
$$

In $3 \mathrm{D}$, this gives $p_{c} \leq 26 / 27$. For anisotropic distributions in different directions, we have 


$$
p_{c} \leq 1-\frac{1}{2^{D}} \prod_{\ell=1}^{D}\left(1-q_{\ell}\right)
$$

where $q_{\ell}$ is the probability of finding a car at a site moving in the $\ell$-th direction provided that there is a car at the site, and thus $\sum_{\ell}^{D} q_{\ell}=1$. As a result, in the infinite dimensional limit, we find that $p_{c} \leq 1$ which is consistent with the mean field theory result that $\langle v\rangle=1-p$ where $p$ is the probability of finding a car in a site.

In summary, we argued that the critical car density has an upper bound less then unity in traffic flow problems in $D>2$.

\section{ACKNOWLEDGMENTS}

This work was initiated while one of us (HFC) was visiting the Chinese University of Hong Kong under the support of a Direct Grant for Research 1994-95 at CUHK. Work at the Institute for Advanced Study was supported by DOE grant DE-FG02-90ER40542. 


\section{REFERENCES}

[1] K. Nagel and M. Schreckenberg: J. Phys. I France 2 (1992) 2221.

[2] T. Nagatani: J. Phys. Soc. Jpn. 62 (1993) 3837; J. Phys. A 26 (1993) 6625.

[3] M. Fukui and Y. Ishibashi: J. Phys. Soc. Jpn. 62 (1993) 3841.

[4] T. Nagatani and T. Seno: Physica A 207 (1994) 574.

[5] K. H. Chung and P. M. Hui: J. Phys. Soc. Jpn. 63 (1994) 4338.

[6] O. Biham, A. A. Middleton and D. Levine: Phys. Rev. A 46 (1992) R6124.

[7] T. Nagatani: Phys. Rev. E 48 (1993) 3290.

[8] T. Nagatani: J. Phys. Soc. Jpn. 62 (1993) 2656; 63 (1994) 1228.

[9] J. A. Cuesta, F. C. Martinez, J. M Morea, and A. Sánchez, Phys. Rev. E 48 (1993) R4175.

[10] K. H. Chung, P. M. Hui, and G. Q. Gu, Phys. Rev. E 51 (1995) 772.

[11] Y. Ishibashi and M. Fukui: J. Phys. Soc. Jpn. 63, (1994) 2882.

[12] H. F. Chau, P. M. Hui and Y. F. Woo: in preparation (1995). 


\section{FIGURES}

FIG. 1. Partition of a two dimensional square lattice by elements containing three sites. The dashed line outline one of the elements. 


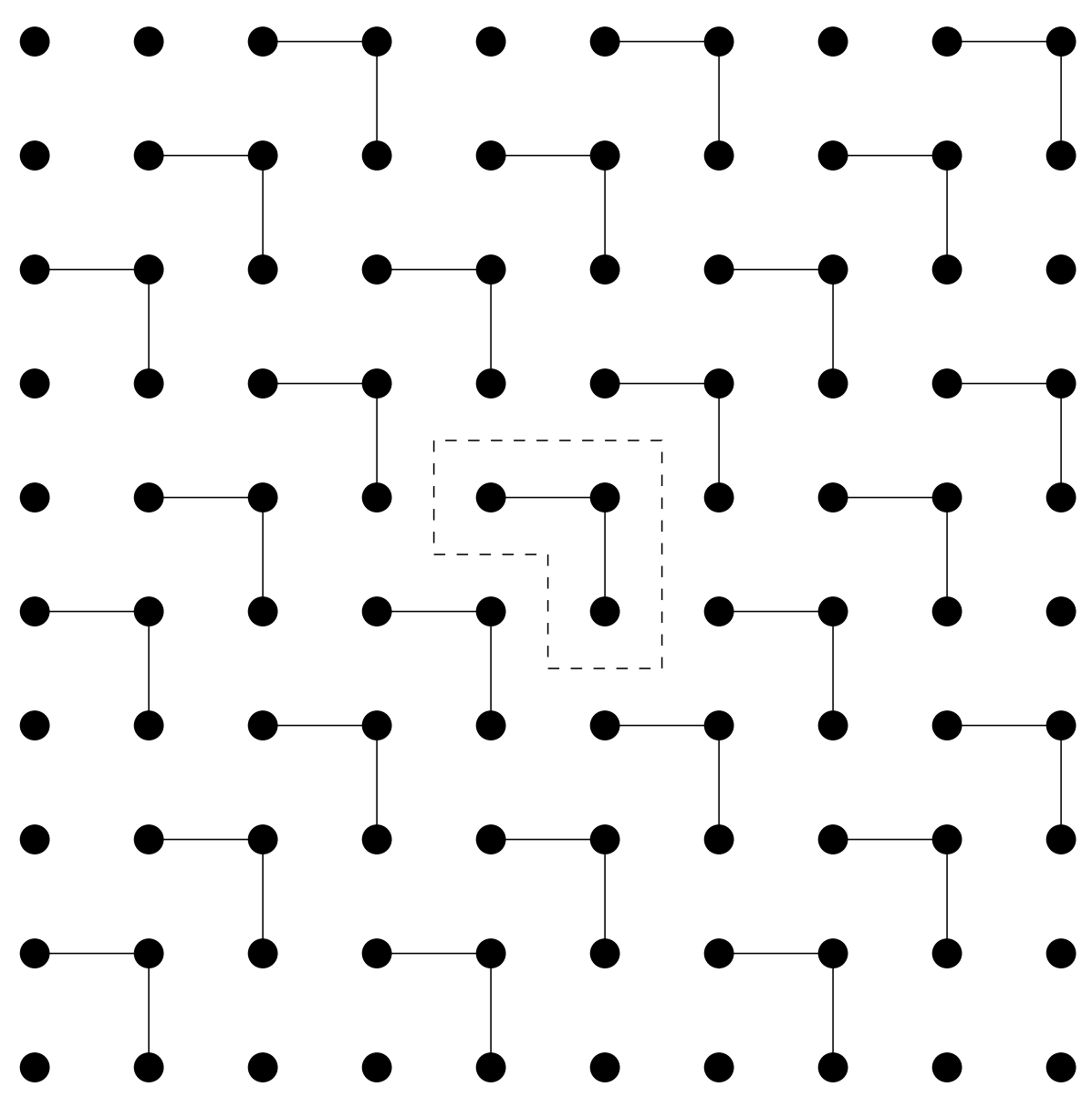

Fig. 1 